\title{
Reflets
}

Revue d'intervention sociale et communautaire

RINFRET-RAYNOR, Maryse, et collab. (2014). Violences envers les femmes : réalités complexes et nouveaux enjeux dans un monde en transformation, Presses de l'Université du Québec, $358 \mathrm{p}$.

\section{John Flynn}

Volume 21, numéro 1, printemps 2015

URI : https://id.erudit.org/iderudit/1032560ar

DOI : https://doi.org/10.7202/1032560ar

Aller au sommaire du numéro

Éditeur(s)

Reflets, Revue d'intervention sociale et communautaire

ISSN

1203-4576 (imprimé)

1712-8498 (numérique)

Découvrir la revue

Citer ce compte rendu

Flynn, J. (2015). Compte rendu de [RINFRET-RAYNOR, Maryse, et collab. (2014).

Violences envers les femmes : réalités complexes et nouveaux enjeux dans un monde en transformation, Presses de l'Université du Québec, 358 p.] Reflets, 21(1), 249-255. https://doi.org/10.7202/1032560ar d'utilisation que vous pouvez consulter en ligne.

https://apropos.erudit.org/fr/usagers/politique-dutilisation/ 


\section{Violences envers les femmes : réalités complexes et nouveaux enjeux dans un monde en transformation}

RINFRET-RAYNOR, Maryse, et collab. (2014). Presses de l'Université du Québec, 358 p.

\section{Par John Flynn}

Violences envers les femmes : réalités complexes et nouveaux enjeux dans un monde en transformation se fonde sur le constat que différentes positions sociales (femmes ayant une incapacité, lesbiennes et transgenres, minorités linguistiques et culturelles, femmes immigrantes, réfugiées ou colonisées) "s'imbriquent et augmentent les vulnérabilités à la violence » (p.VII).Ainsi, chercheuses, chercheurs, intervenantes, intervenants et diverses personnes jouant un rôle politique ou administratif dans l'offre de services ont été invités à travailler de concert pour mieux comprendre les contextes spécifiques où ces violences se manifestent et pour élaborer des stratégies de prévention et de soutien aux femmes qui les subissent. Cet ouvrage collectif a été réalisé à la suite du $2^{\mathrm{e}}$ colloque international du Centre de recherche interdisciplinaire sur la violence familiale et la violence faite aux femmes (CRIVIFF) tenu à Montréal au printemps 2011.

L'ouvrage se décline en dix-sept chapitres regroupés en trois parties ayant pour thèmes les perspectives théoriques et de recherche, les contextes de vulnérabilité, ainsi que les effets et les limites des interventions sur les plans politique, juridique et psychosocial. 


\section{Partie 1 - Violences faites aux femmes : de nouvelles perspectives théoriques et de recherche}

Cette première partie de l'ouvrage se divise elle-même en trois parties qui ont pour titres : Violence conjugale - Dimension interpersonnelle (chapitres 1 et 2), Violences faites aux femmes Dimensions structurelles (chapitres 3, 4 et 5) et Violences faites aux femmes - Examen des facteurs de risque (chapitres 6 et 7).

Le chapitre 1 signé Johnson et le chapitre 2 signé Stark portent sur de nouvelles théories visant à mieux comprendre les différentes dynamiques interpersonnelles des violences qui existent au sein de certains couples et leurs effets sur les victimes. Johnson discute de l'importance de distinguer les types de violence - terrorisme intime, résistance violente, violence situationnelle au sein d'un couple - sans quoi nous risquons de tirer des conclusions erronées et de mettre en place des interventions inadéquates. C'est le cas, par exemple, lorsqu'une situation de résistance violente est faussement identifiée comme en étant une de terrorisme intime, ce qui contribue d'ailleurs à renforcer la perception d'une symétrie de la violence entre les genres. Selon Johnson, la différence entre ces types de violences se situe dans les rapports de force et dans la dynamique de domination générale opérant au sein de la relation et non dans la nature des attaques. Stark, pour sa part, discute des conséquences de l'adoption quasi universelle d'un modèle de violence axé sur les violences physiques graves. Ce modèle "dissimule la nature historique des abus, fragmente et banalise l'oppression subie " et "ignore les nombreuses tactiques non violentes, typiques de la plupart des cas dans lesquels les femmes victimes sollicitent une aide extérieure, et qui sont souvent plus préjudiciables et plus prépondérantes que les agressions » (p. 3435). Ainsi, Stark propose un modèle alternatif féministe qui se concentre sur le "contrôle coercitif " exercé par les agresseurs et sur les tactiques qu'ils utilisent pour dominer et isoler leur conjointe et pour brimer sa liberté. 
Les dimensions structurelles des violences faites aux femmes font l'objet de trois chapitres qui proposent chacun une discussion réflexive sur différentes théories féministes. Au chapitre 3, Anthias, propose une analyse intersectionnelle des violences envers les femmes. L'auteure rappelle qu'il est fondamental d'écouter les récits des femmes violentées sans toutefois oublier de se concentrer sur le paysage social et économique plus large. En effet, elle soutient que "l'attention doit être portée à la fois sur l'expérience, nous informant de l'agentivité et de l'identité sur les structures de pouvoir» (p. 72). Dans son étude portant sur l'expérience de femmes immigrantes proposée au chapitre 4, Harper privilégie l'approche narrative. Donner une voix à ces femmes tout en créant des espaces où elles peuvent être entendues permet une réappropriation des problématiques qui les touchent, ce qui résulte dans l'élaboration de stratégies mieux adaptées à leurs besoins. Enfin, au chapitre 5, Weinstock lance un appel à la réconciliation entre les féministes et les multiculturalistes progressistes en démontrant comment ces deux approches se complètent plus qu'elles ne s'opposent. Pour y parvenir, l'auteur désavoue un multiculturalisme okinien - "selon Okin, la protection des droits groupes culturels minoritaires va à l'encontre de l'intérêt des femmes.» (p. 103) — au profit d'un multiculturalisme dynamique, reconnaissant la capacité de toutes les femmes d'agir en tant qu'agentes de changements culturels.

Cette première partie de l'ouvrage se termine sur une évaluation des facteurs de risque, laquelle vise à mieux prédire des situations où des femmes vivent de la violence conjugale. Ouellet et Cousineau au chapitre 6 et Dubé et Drouin au chapitre 7 montrent que l'analyse systématique de données et l'utilisation des méthodes mixtes permettent de mesurer divers facteurs qui influent sur le parcours des femmes victimes de violence, par exemple le rôle des maisons d'hébergement ou la détention du partenaire violent. L'analyse présentée dans ces deux chapitres permet de prédire avec une plus grande justesse les contextes dans lesquels la violence conjugale risque d'émerger. 


\section{Partie 2 - Des réalités complexes et des contextes de vulnérabilité à découvrir}

Aux dires de Rinfret-Raynor et de Lesieux (p. 7), les textes de cette partie permettent « de découvrir la multiplicité des réalités vécues par les femmes victimes de violence selon des étapes particulières de vie et selon différents contextes " afin de " rendre compte de la spécificité des expériences de victimisation et des mécanismes sociétaux susceptibles de vulnérabiliser les femmes ».Au chapitre 8 , Fernet et collab. se penchent sur les sources de conflits dans les couples adolescents et sur les stratégies que les adolescentes et adolescents utilisent pour y remédier. Le manque de confiance en l'autre et la jalousie seraient les plus grandes sources de conflits pour ce groupe d'âge. Les entrevues menées auprès de nombreux couples d'adolescents démontrent que les stratégies de gestion de conflits « oscillent entre la communication ouverte et la violence » (p. 159) et les résultats de l'étude soulignent l'importance de la prévention, de la sensibilisation et de la promotion d'habiletés saines en matière de gestion de conflits.

Signé Fortin, Dugal et Guay, le chapitre 9 porte sur les situations de violences au sein de jeunes couples adultes (18 à 25 ans). Plusieurs études démontrent que les jeunes couples sont plus à risque de vivre des violences que ceux plus âgés et que ces violences sont d'ordre " situationnel " plutôt que de terrorisme intime. Les auteurs du chapitre discutent des conséquences psychologiques pour les jeunes adultes qui sont victimes de violence conjugale et expliquent comment ils oscillent entre les ressources d'aide formelle et les ressources informelles, selon la gravité des violences subies.

Tremblay aborde au chapitre 10 une problématique sociale encore fort peu étudiée, soit celle des violences commises à l'endroit des aînées. L'auteure soulève plusieurs contextes de vulnérabilité dans lesquels vivent ces femmes, tels l'isolement, le manque d'informations et l'âgisme. Elle décrit également un projet québécois élaboré à l'intention de personnes âgées autochtones 
et non autochtones victimes de violences sexuelles et ayant pour objectifs la sensibilisation et la formation, l'autodétermination et le transfert de connaissances.

Enfin, Tutty et collab. présentent au chapitre 11 les résultats d'une étude menée auprès d'une soixantaine de femmes sans domicile fixe et mettent en lumière les défis spécifiques auxquels elles sont confrontées et les violences structurelles rattachées à leurs réalités. Drif et Touami consacrent le chapitre 12 à la prévention des mariages forcés, tandis que Bigstone et collab. traitent au chapitre 13 des résultats d'un projet pilote, Walking the path together, destiné à l'accompagnement prolongé (selon une approche culturellement adaptée) de femmes et d'enfants autochtones ayant été victimes de violence.

\section{Partie 3-Les réponses politiques, judiciaires et psychosociales aux violences faites aux femmes}

Différentes formes d'intervention, soit politiques, judiciaires et psychosociales, forment l'essentiel de cette partie de l'ouvrage. Lesieux, Rinfret-Raynor et Brodeur présentent au chapitre 14 les services en maisons d'hébergement pour femmes victimes de violence conjugale au Québec de 2004 à 2009. Y sont aussi abordées les grandes étapes de la politique québécoise en matière de violence conjugale et les initiatives du gouvernement québécois depuis les quarante dernières années, lesquelles ont culminé en 1995 avec la Politique d'intervention en matière de violence conjugale, laquelle a mené à la reconnaissance de la violence conjugale en tant que prise de pouvoir et non perte de pouvoir.

Au chapitre 15, Smedslund compare les approches utilisées par trois états européens - l'Allemagne, l'Autriche et l'Espagne - pour lutter contre ces violences et discute des défis spécifiques auxquels ces états étaient confrontés lors de leur mise en œuvre de pratiques novatrices dans leur lutte contre les violences faites aux femmes. Harris Kluger consacre le chapitre 16 aux mesures législatives américaines développées pour mieux protéger les 
femmes victimes de violence conjugale. Malgré ces améliorations, plusieurs défis persistent, notamment pour les nouvelles arrivantes et les personnes vivant en situation de handicap. Enfin, au chapitre 17, Gauthier et collab. prônent l'approche de la réduction des méfaits chez les intervenantes et intervenants œuvrant auprès des femmes victimes de violence conjugale qui choisissent de ne pas quitter leur partenaire violent. Les auteures anticipent les bénéfices d'une telle approche en violence conjugale et analysent sa compatibilité avec l'approche féministe.

\section{Appréciation critique et conclusion}

Les auteures et auteurs de Violences envers les femmes : réalités complexes et nouveaux enjeux dans un monde en transformation démontrent une bonne compréhension des enjeux et des défis afférents à l'intervention auprès des femmes victimes de violence conjugale. La richesse de cet ouvrage collectif se trouve dans la multiplicité des contextes étudiés, notamment ceux des femmes immigrantes et des femmes autochtones, afin de mieux comprendre la victimisation des femmes, ainsi que des pistes d'interventions et les politiques devant être mises en place pour y remédier. Un autre tour de force de cet ouvrage réside dans le fait que plusieurs groupes d'âge (des adolescentes aux aînées) font l'objet d'études, permettant de mieux identifier les enjeux et défis d'intervention qui sont propres à chacun.

Toutefois, il aurait été intéressant d'accorder plus de place aux récits personnels des femmes victimes de violence. Également, il aurait été intéressant de recueillir des témoignages d'enfants exposés à la violence conjugale - tout en reconnaissant les difficultés que cela pourrait poser - et de consacrer une partie de l'ouvrage aux conséquences qu'a sur eux la violence conjugale.

En conclusion, cet ouvrage dresse un bilan vraisemblablement exhaustif des connaissances concernant la problématique, tout en offrant plusieurs pistes novatrices intéressantes pour l'intervention. Le contenu de l'ouvrage est accessible et sa lecture sera bénéfique à toute personne désireuse de contribuer à la lutte contre ce sexisme 
structurel, autant aux femmes victimes de violence conjugale qu'aux étudiantes, étudiants, professeures et professeurs en études des femmes ou en service social, intervenantes, intervenants et à toute personne jouant un rôle politique ou administratif dans l'offre de services. 